\title{
QoS-Aware Video Communications over TDMA/TDD Wireless Networks ${ }^{\star}$
}

\author{
Francisco M. Delicado, Pedro Cuenca, and Luis Orozco-Barbosa \\ Albacete Research Institute of Informatics \\ University of Castilla la Mancha \\ Campus Universitario s/n, 02071 Albacete, Spain \\ \{franman, pcuenca, lorozco\}@info-ab.uclm.es \\ Tlf.: +34-967-599200 ext 2497 \\ Fax: +34-967-599224
}

\begin{abstract}
In recent years there has been an explosive growth on the use of wireless video communications. Despite much research in this field, the deployment of effective QoS-aware real-time video services over wireless channels remains a challenging task. In this paper, we first introduce and describe an overall system architecture capable of offering true end-to-end QoS guarantees to MPEG-4 video services running over TDMA/TDD wireless networks. The proposed system architecture is built by integrating two key system elements: a set of control mechanisms and various error resilient techniques. After reviewing the various system elements, we evaluate the use of the various mechanisms. We show the effectiveness of the proposed architecture in terms of various metrics. Our results show that the video quality as perceived by the end user can be significantly improved by making use of hierarchical video coding techniques.
\end{abstract}

Keywords: TDMA/TDD, WLAN, QoS, Hierarchical Video Coding, Multimedia Communications, Video Quality.

\section{Introduction}

In recent years, we have been witnessing an increasing interest in deploying wireless video communications. Despite much research in this field, the provisioning of QoS guarantees to real-time video over wireless channels remains a challenging task. Two major issues in providing true end-to-end wireless video capabilities are: mechanisms enabling the provisioning of QoS guarantees and the robustness of video compression algorithms operating over error-prone environments. From the point of view of the network, the main challenge when using VBR coded video is to guarantee the required quality of service (QoS). The deployment of

\footnotetext{
* This work was supported by the Ministry of Science and Technology of Spain under CICYT project TIC2003-08154-C06-02 and the Council of Science and Technology of Castilla-La Mancha under project PAI-06-0106.
} 
proper control mechanisms both within the TDMA/TDD protocol architectures and the video application are needed to enable the deployment of effective digital video applications over wireless networks. This is due to the changing demands on resources, e.g., channel capacity and buffer space, needed for the transport of the video streams across the network as well as to the wireless channel characteristics. In order to overcome some of these problems, a set of control mechanisms have to be introduced into the protocol architecture of video communication systems.

Regarding the video application, video compression algorithms inherently remove redundancies making it more difficult to decode information under error and loss conditions. Major video coding standards make use of variable length code words and predictive frame coding aiming to significantly improve efficiency but at the cost of robustness. With variable length coding, packet losses can cause the decoder to incorrectly determine the length of a codeword leading to loss of synchronization in the decoding process. Since predictive coding techniques encode only the differences between frames or macroblocks, losses are much likely to propagate through the video stream thus degrading video quality. The effects of a packet loss will persist until non-predictively coded information occurs in the stream. In this work, we then show how error resilience techniques used in video compression algorithms can be used to improve the video quality. We argue that the final video quality perceived by the user can be significantly improved by the inclusion of hierarchical video coding techniques by sending the data into two separate streams. This allows the application to request various QoS guarantees from the video service, i.e., enabling the transmission of more sensitive data via a more reliable service.

We evaluate the effectiveness of our proposals in terms of the following metrics: network throughput, overhead, jitter, fairness and packet losses. A distinctive feature of our study is that we also validate the effectiveness of our proposed schemes by quantitatively evaluating the video quality as perceived by the end user.

The article is organized as follows. Section 2 provides a short overview of the operation of TDMA/TDD networks and describe a complete set of QoS mechanisms introduced in one of our previous work [1. These mechanisms aim to provide the QoS guarantees required by time constrained applications when coexisting with other services. Section 3 describes techniques included in the MPEG-4 standard to increase robustness into the encoded video streams. A description of a hierarchical video coding technique for MPEG-4 is shown in this section. The results of our performance evaluation study are given in Section 4 . Finally, Section 5 concludes the paper.

\section{TDMA/TDD QoS-Aware Mechanisms}

\subsection{Principles of TDMA/TDD Networks}

In a TDMA/TDD network the communications between all components of the networks is organized in frames. In each frame, the Base Station (BS) allocates 
the time slots in response to the previous Subscriber Station (SS) requests. In this way, each SS has to request the required resources from the BS by issuing a Resource Request (RR) message, while the BS informs the SS of the positive outcome by using a Resource Grant $(R G)$ message.

A normal TDMA/TDD frame is divided into four fundamental phases:

Broadcast phase: this phase is used to carry out the overall frame control information. It contains the configuration parameters of the downlink and uplink phases, such as the positions and number of resources allocated to each active connection in each transmission phase. It is in this map that a control messages is typically included to convey the outcome of a successful resource request, i.e., a resource grant (RG) message.

Downlink phase: it is the portion of frame which carries user data and control connections messages from the BS to the SS's.

Uplink phase: similar to the downlink phase, this phase is formed by the user data and control messages associated to the uplink connections.

Random Access phase: it is a portion of the frame which can be accessed by all SS's using a contention process. This phase could be used by stations which have not been granted resources in the current frame to place their resource requests.

\subsection{Resource Request Mechanisms}

In one of our previous works [1], we have specified the following four different resource request mechanisms taking into account the QoS requirements of various types of applications:

Type 1: This mechanism is based on a contract. The contract is established after negotiation between an SS and the BS, at setup time. During this initial negotiation, the amount of resources to be granted to the SS are set. This type of resource request mechanism is suitable for applications characterized by a constant bit rate, such as, CBR-encoded voice services.

Type 2: Under this second type, the resource request mechanism is initiated by the BS through a polling mechanism. The BS polls the SS at the beginning of the connection allowing the SS to request the amount of resources it requires. The BS then polls once again the SS after having fulfilled the previous request or after a given time interval from the previous poll, whenever happens first. The length of the interval between polls should be set accordingly to the needs of the application.

Type 3: Under this request mechanism, the SS has to request its resources by sending a message using a contention process. Once having finished the allocation of the resources required by the SS, the BS, similarly to the Type 2 mechanism, polls the SS.

Type 4: The main difference between this type with respect to the Type 3 request mechanism comes from the fact that regardless of the activity of the connection, the SS has to go through a contention process in order to place 


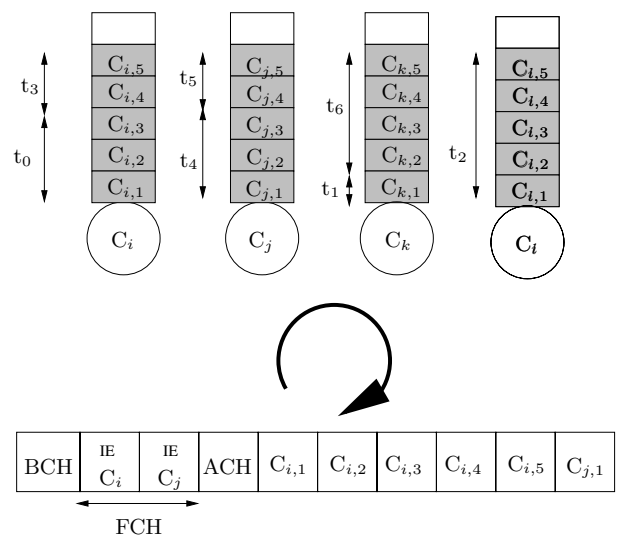

Fig. 1. Bandwidth allocation scheme operation

its resource request. In particular, different to the previous type, Type 3, once the BS has finished fulfilling the SS requirement, the SS has to go once again through a contention cycle to place its request.

\subsection{Bandwidth Allocation Schemes}

The BS has as one of its main duties the implementation of the actual bandwidth allocation scheme. Since there is one message in the broadcast phase per each connection, the amount of overhead introduced into the frame will heavily depend on the way the resources are assigned to the various connections. However, this allocation should not penalize the ability of providing the QoS requirements to the various applications.

These two factors: the amount of overhead introduced for the mechanism to operate and the traffic differentiation capabilities were studied by us in [2]. In that study, we introduced a novel scheme addressing these two issues, namely, the Minimum Overhead Round Robin (MORR) scheme. The main aim of the MORR scheme is to limit the amount of overhead to be introduced in the frame by contiguously allocating the channels to a given connection. The mode of operation of the MORR mechanisms is depicted in Figure 1, where $\left(\mathrm{t}_{i}\right)$ denotes the arrival time of the requests, and a queue is assigned to each one of the active connections. For the purpose of this work, we will further enhance this scheme by integrating a two-level priority policy at each queue. This policy should be particularly useful when dealing with hierarchical encoded video streams.

\section{MPEG-4 Error Resilience Tools}

As already stated, the fact that the MPEG-4 video coding scheme uses compression techniques makes any MPEG-4 based video communications application very vulnerable to packet losses. In the absence of any error propagation control 
mechanism, the loss of each unit of information may cause the loss of information up to the next synchronization point, e.g., Video Object Plane-VOP headers. In other words, a packet loss in the coded video bit-stream will result on the loss of all the macroblocks that follow up to the end of the current VOP. This phenomenon is known as spatial impairment propagation. Furthermore, due to the predictive nature of the MPEG-4 algorithm, when errors or losses occur in an I or P-VOP, the VOPs encoded using as reference the affected I or P-VOP will not be properly decoded. The losses will propagate until the next intra-coded VOP; this is referred to as temporal impairment propagation.

To address these issues the MPEG-4 video standard defines a set of special error resilience tools 3 . The standard supports flexible re-synchronization markers and data partitioning features to separate motion and header information from texture information and reversible variable length coding.

\subsection{Video Packet Resynchronization (VP)}

Video packet resynchronization is an approach aiming to reduce the spatial propagation of errors. This approach consists in introducing resynchronization markers into the bit-stream. Whenever the decoder detects an error, it can then look for the following resynchronization marker and quickly regain resynchronization. According to the specifications of the MPEG-4 standard, the resynchronization markers can be periodically inserted every $\mathrm{K}$ bits; this scheme divides the bitstream into data packets that are independent from each other named Video Packets (VP). At the beginning of each video packet, the encoder inserts two additional fields in addition to the resynchronization markers to remove all data dependencies between the data belonging to two different video packets. These are: the absolute macroblock number of the first macroblock in the video packet (MB number), which indicates the spatial location of the macroblock in the current VOP; and the quantization parameter $\left(Q_{p}\right)$ used to quantize the DCT coefficients.

\subsection{Data Partitioning (DP) - Hierarchical Video Coding}

The Data Partitioning (DP) error resilience tools defined by the MPEG-4 standard specifies that each I or P-VOP video packet can be divided into two different partitions. The first one (the most important part of the video packet) contains the header, which encodes the information pertaining to the first macroblock of the video packet and the HEC extension, if used, shape data, the motion information (for P-VOP's) or DC coefficients (for I-VOP's) of the macroblocks. The second partition contains the AC's coefficients of the macroblocks, for I-VOP's, and the texture information for $\mathrm{P}^{-\mathrm{VOP}^{\prime}}$ s. If the second partition is lost during transmission, the MPEG-4 decoder can decode the video packet using only the first partition. In [4, we have shown that extending this hierarchical encoding scheme to the B-VOP's proves effective in enhancing the robustness of the video encoded stream. We then consider its use in this work. 


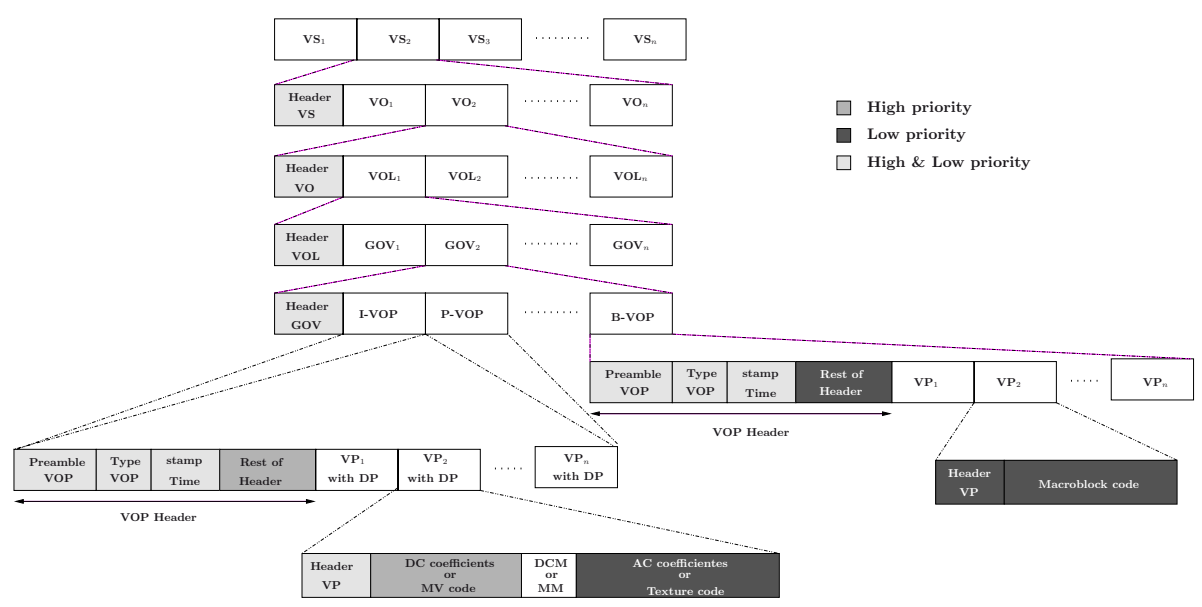

Fig. 2. Hierarchical MPEG-4 Video Coding Scheme proposed

In order to actually transmit the encoded video stream using the DP tools, we prioritize the transmission of the first partition over the second one. Towards this end, we will consider that the aforementioned bandwidth allocation mechanisms implement a priority discipline. In this way, whenever a high priority packet (pertaining to the first partition) be present in the output queue, this one will be first sent over the wireless link. This mechanism should reduce the possibility of losing high-priority video packets due to excessive delays in accessing the channel. However, in order for the decoder to be able to reconstruct the video stream, we have to include the VOP start code, the type of VOP and the time stamps into the second partition; the low priority video stream. To ensure synchronization within VOP, the resynchronization marker of the video packet and the number of the first macroblock have also to be copied into the video packet of the lowpriority partition. It should be clear that this scheme, referred from now on as Hierarchical Video Coding, adds some extra overhead traffic by requiring the duplication of some parts of the video data. The actual amount of overhead will depend on the particular setup characteristics. In the system being considered throughout our simulations, the overhead introduced by adding this feature is approximately $1.2 \%$ of the total video data with respect to the non-hierarchical encoded video.

\subsection{Reversible Variable Length Codes (RVLC)}

RVLC can be used in conjunction with the aforementioned data partitioning tools to recover more DCT data from a corrupted texture partition. RVLC's are designed such that they can be decoded both in the forward and in the backward direction. If the decoder detects an error while it is decoding the texture part in forward direction, it looks for the next resynchronization marker (start of the 
next video packet), and then decodes the texture part in the backward direction until an error is detected. Based on the two errors location, the decoder can recover some of the data that would have otherwise been discarded.

\section{Performance Evaluation}

In order to evaluate the proposed mechanisms, we have developed a simulator using OPNET 10.0 [5]. This simulator has been developed using the frame structure of HIPERLAN/2. In our simulations, we use one HIPERLAN/2 cell operating in centralized mode supporting four types of service: voice, video, besteffort and background. In the composition of the frame we use short preambles, guard times of $2 \mu \mathrm{s}$, the random access phase is formed by three transmission slot and the physical mode for the control and data user messages are QPSK-3/4 (18 Mbps) and 16QAM-9/16 (27 Mbps), respectively.

Given that one of the main objectives of this study is to evaluate the performance and effectiveness of the proposed system architecture, we have considered out two main scenarios. Under the first scenario, namely Scenario without QoS, all applications have to go through a contention-based process when attempting to transmit each and every resource request packet. Under the second scenario, Scenario with QoS, each of the applications makes use of a different type of mechanism. The following has been used: voice services make use of the Type 1 mechanism with 48 bytes reserved every 12 frames (this corresponds to a guaranteed data rate of $16 \mathrm{Kbit} / \mathrm{s}$ ). Video services make use of the Type 2 mechanism with a timer period of $40 \mathrm{~ms}$. The value of this latter parameter has been derived based on the results obtained in our previous studies. The best-effort (BE) and background traffic (BK) make use of the Type 3 and Type 4 mechanisms, respectively.

The voice traffic is implemented using a constant bit-rate voice source encoded at a rate of $16 \mathrm{Kbits} / \mathrm{s}$ according to the G.728 standard [6]. The voice sources are randomly activated within the first $24 \mathrm{~ms}$ of the simulation. The video traffic has been characterized by MPEG-4 [3] video traffic traces. Each video application begins its transmission within a random period given by the expression $t=$ uniform $\left(0, \frac{12}{f}\right)$ being $f$ the video frame rate. In this way, the peak periods of the source rates are randomly distributed along a Group Of Pictures (GOP) period. The transmission of a video frame is uniformly distributed along the interval of duration of a frame $\left(\frac{1}{f}\right)$. We use the sequence Funny encoded on CIF format at 25 frames/s. The video sequence has been encoded using the DP scheme and integrating the RVLC scheme with Video Packets of 768 bits. We consider, both, the transmission of the video sequence using the same priority levels for the two partitions, referred as the non-hierarchical case, and by using a hierarchical transmission scheme, i.e., two levels of priority. The best-effort traffic is generated using the traffic model for Web surfing applications described in [7]. The background traffic generated by each source is a combination of FTP, e-mail and Napster according to the model described in [8]. The traffic sources of these two latter traffic types are initiated at the beginning of the simulation run. 
All connections are assumed to be running in both directions, i.e., uplink and downlink. In order to carry out this study, we have considered that one third of the SS's will be running voice/video applications. Another third of the SS's generate best-effort traffic and all the other SS's generate background traffic. We start by simulating a wireless network consisting of three SS's. We then gradually increase the Total Offered Load of the wireless LAN by increasing the number of SS's by three. In this way, the stations are always incorporated into the system in a ratio of 1:1:1 for voice/video, best-effort and background, respectively. We increase the number of terminals on a three by three basis starting at 3 and up to 18 stations. In this way, the normalized offered load is increased from 0.16 up to 1.2. We have preferred to evaluate a normalized offered load, rather the absolute value. The normalized offered load is determined with respect to the theoretical maximum capacity (27 Mbps).

\subsection{Metrics}

In our study, we have been interested in assessing the performance in terms of the following metrics: total normalized throughput, overhead, jitter distribution, packet loss rates, packet loss distribution, fairness and video quality.

The analysis of the total normalized throughput shows the utilization of the wireless medium. This metric refers to the percentage of the total offered data (the traffic from all the sources) that is actually delivered to the destination. It should be clear that this metric lies within the interval $[0,1]$. When this metric is less than 1 , this fact indicates us that the presence of packet losses.

In order to provide us a clear indication of how the capacity of the channel is being used, the overhead metric is evaluated. It is a relative measure and it is simply defined as the ratio between the control bits and the total number of bits (data plus control) being sent, i.e., composing the frame. It should be clear that at low loads, there may be some spare capacity, i.e., the frame is not completely filled up.

In order to limit the delay experienced by the voice and video applications, an essential condition to guarantee the QoS required by both applications, the maximum time that a unit of voice and video may remain in the transmission buffer has been set to $10 \mathrm{~ms}$ and $100 \mathrm{~ms}$, respectively. These time limits are in line with the values specified by standards and in literature 9. A packet exceeding this upper bound is dropped. The loss rate due to this mechanisms is given by the Packet Loss Rate (PLR).

An important measure when evaluating packet loss rates for applications particularly sensitive to the packet loss, like MPEG-4 video compressed applications, is the length of a loss burst $\left(L_{\text {burst }}\right)$ and the distance between bursts $\left(D_{\text {burst }}\right)$. We evaluate this loss distribution using the CDF. It is well known that the quality of the video sequence heavily depends on the loss pattern. In particular, a long burst will make it practically impossible for the decoder to recover the information. On the contrary, in the presence of short loss bursts, a decoder may be able to reconstruct part of the lost information through the use of the RVLC scheme. 
One of the most important metrics in multimedia communications is the quality of the received video sequence. This has been evaluated using the Moving Picture Quality Metric (MPQM) [10]. This metric has proved to behave consistently with the human judgments, i.e., according to the quality scale that is often used for subjective testing in the engineering community (see Table 1).

Table 1. Video Quality Scale

\begin{tabular}{c|c|c}
\hline \hline Rating & Impairment & Quality \\
\hline 5 & Imperceptible & Excellent \\
\hline 4 & Perceptible, not annoying & Good \\
\hline 3 & Slightly & Fair \\
\hline 2 & Annoying & Poor \\
\hline 1 & Very annoying & Bad \\
\hline \hline
\end{tabular}

Finally, we evaluate the fairness of the allocation schemes between flows of the same type (voice, video, best-effort and background). For this purpose, we calculate the Fairness Index (FI) defined in [11. This index is defined as:

$$
F I=\frac{\left(\sum_{i=1}^{n} T_{i}\right)^{2}}{n \times \sum_{i=1}^{n}\left(T_{i}\right)^{2}},
$$

where $n$ is the number of the same type flows, and $T_{i}$ is the throughput of the flow $i$. Recall that $F I \leq 1$, and it is equal to 1 if all $T_{i}$ are equal, which corresponds to the highest degree of fairness between the different users.

In the simulation results, each point in the plots is an average over forty simulation runs, and the error bars indicate the $90 \%$ confidence interval. Moreover, our measurements started after a warm-up period allowing us to collect the statistics under steady-state conditions.

\subsection{Simulation Results}

Figure 3 represent the normalized (carried) throughput by type of traffic as a function of the offered load for both scenarios, with and without QoS, and in the case of use QoS using two allocation algorithms, FIFO and MORR. For voice traffic, Figure 3. (a), it is appreciated that in the scenario with QoS, all the traffic is served independently of the load. This is due to the fact that the Type 1 request method consists in contract all the resources need for this connections. In the case when all the requests are sent using a contention process, the performance decreases rapidly as soon as the load exceeds $50 \%$ of the network capacity.

The worst performance for Throughput of video traffic is obtained for the case of the scenario without QoS, Figure 3. (b). The priorization of video request using the Type 2 request mechanism proves effective, particularly when using the MORR scheme. The figure shows that all the video traffic can be effectively served up when the MORR allocation scheme is used. The MORR proves 
more effective in differentiating the video traffic. Figures 3. (c) and (d), represent the throughput for the best-effort and background traffic, respectively. The performance is very similar for all system configuration, except for the slight penalization suffered by the background traffic at high network loads when using the QoS mechanisms. This is due to the fact that the control schemes effectively favor the video traffic over the lowest priority traffic, i.e., the background traffic.

Figure 4 depicts the overhead as a function of the offered load for the three bandwidth allocation schemes under study. As seen in the figure, the overhead decreases as the load is increased for all cases and for loads up to 50\%. For the case of the FIFO mechanism, the overhead introduced in the frame is lower under Scenario without QoS than in Scenario with QoS. This difference is due to the mechanism used to place the requests and the policy used to serve the requests. Remember that under Scenario without QoS, the SS 's make use of a contentionbased process to place their requests. As the load increases, the SS's spend more time attempting to place their requests. As the number of channels requested is being updated during this period of time, a larger number of channels will be requested. Furthermore, since the requests are served following a FIFO policy, the overhead decreases as the number of actual channels used to convey user data is increased.

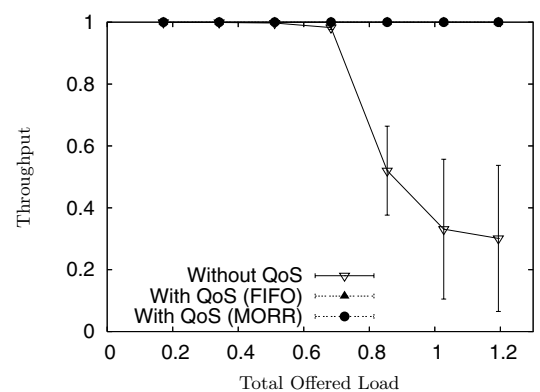

(a) Voice Normalized Throughput

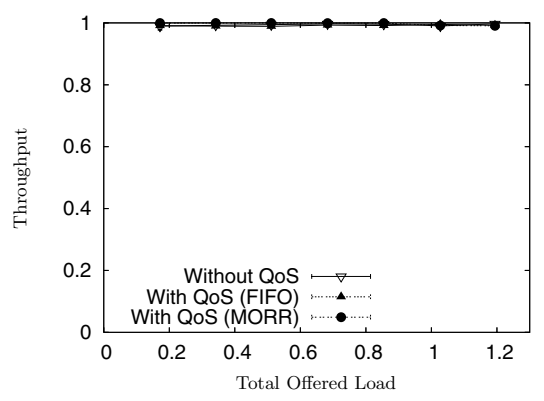

(c) BE Normalized Throughput

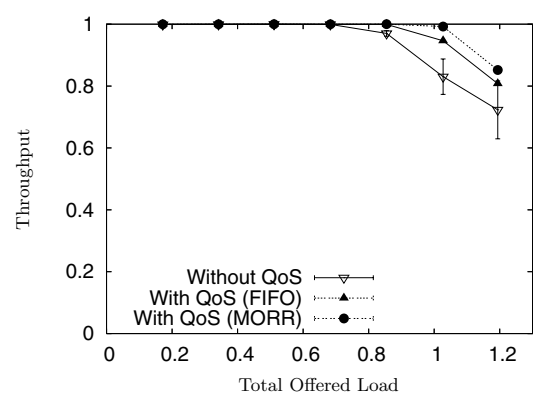

(b) Video Normalized Throughput

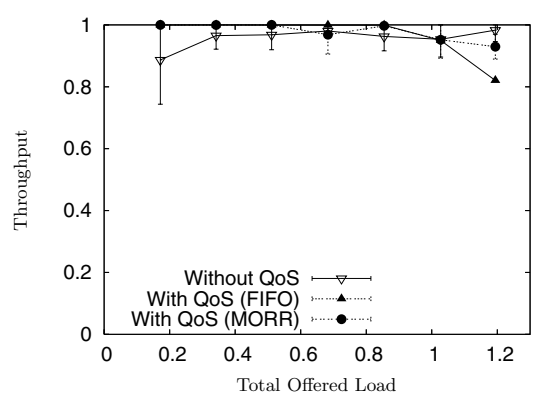

(d) BK Normalized Throughput

Fig. 3. Normalized Throughput for all Connection Types 


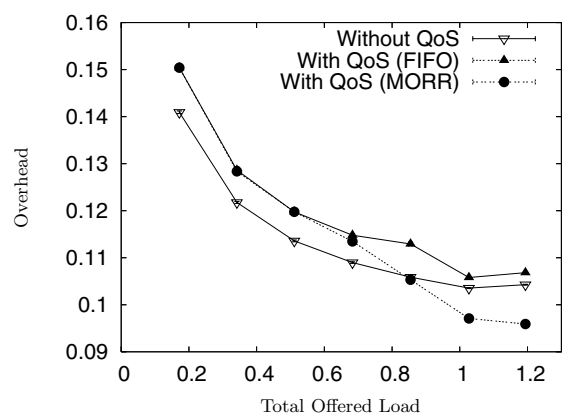

Fig. 4. Overhead

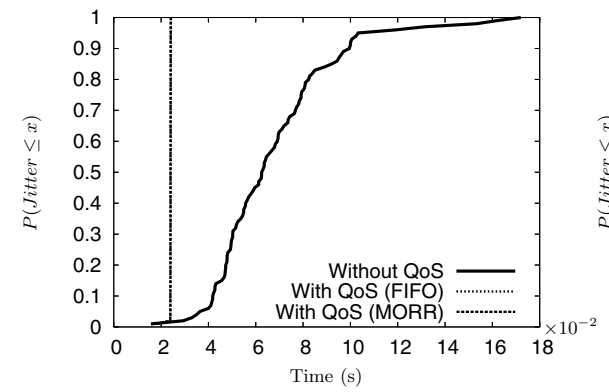

(a) CDF - Voice Jitter.

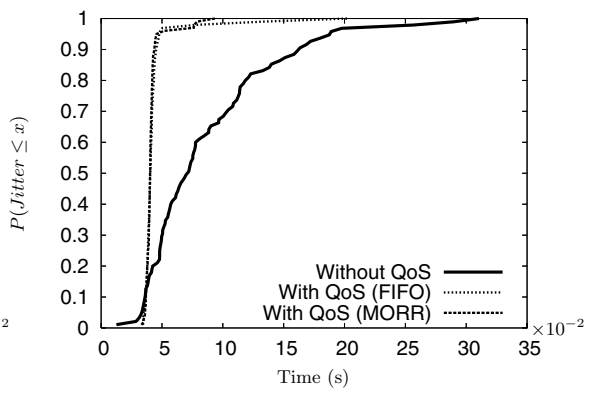

(b) CDF - Video Jitter.

Fig. 5. CDF of Jitter for Voice and Video Connections (Offered Load $\simeq 100$ )

Figure 4 shows that the system combining the contention based procedure and the bandwidth allocation scheme based on a simple FIFO scheme (Scenario without QoS) provides the best results for loads up to $80 \%$. However, as the system is exposed to higher loads, the use of a contentionless process and a more intelligent bandwidth allocation scheme proves to be more efficient.

Regarding the jitter, Figure 5. (a) shows that voice communications do not suffer any deviation since a static allocation of resources ensure the isochronous transmission of the voice packets, one voice packet every $24 \mathrm{~ms}$, independently of the network load conditions.

In the case of the video traffic (Figure 5.(b)), the jitter remains constant for Scenario with QoS when using FIFO and MORR mechanisms. The figure shows that $95 \%$ of the inter-arrival times between frames are $40 \mathrm{~ms}$. This corresponds to the sampling rate of 25 frames $/ \mathrm{s}$, i.e., a frame every $40 \mathrm{~ms}$. This is an excellent result that indicates clearly the effectiveness of the proposed mechanisms.

The Fairness Index of voice and video is shown in Figure 6. In this case, Scenario without QoS shows the worst performance results mainly due to the use of a contention process. In the case of Scenario with QoS, there are not differences between the results obtained using the different allocation schemes under study. 


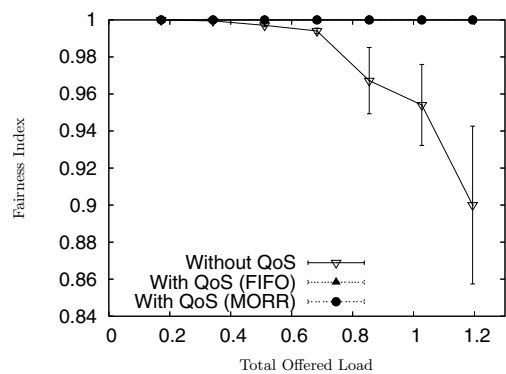

(a) Fairness Index of Voice.

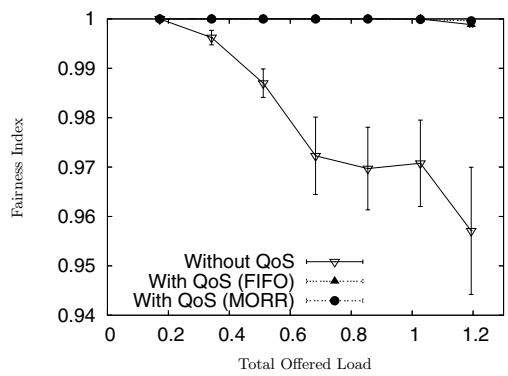

(b) Fairness Index of Video.

Fig. 6. Fairness Index of Voice \& Video

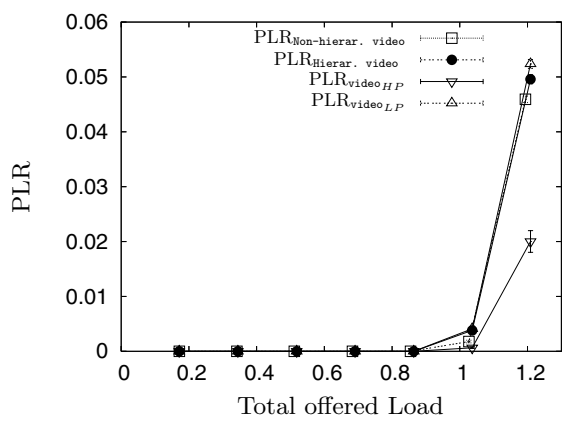

Fig. 7. PLR - Normal and Hierarchical video

To evaluate the performance of the hierarchical video coding, we have used a normal video stream and a hierarchical version of the same sequence and making use of the MORR allocation schemes. We denote by video ${ }_{H P}$ the high priority layer stream of the hierarchical video, and video ${ }_{L P}$, the low priority video layer stream. Figure 7 depicts the loss rates for the normal and hierarchical encoded video. The figure also distinguishes between the packet loss rates of the low and high priority layers of the hierarchical video stream. Obviously, the PLR of the overall hierarchical video is higher than the PLR of the normal video, since the hierarchical process introduces an overhead of $1.19 \%$ into the output stream. As expected, the results show that the low priority video exhibits a higher loss rate when compared to the loss rate experinced by the high priority traffic.

Figure 8. (a) shows the CDF of the size of loss bursts. From the results, it is clear that the loss burst exhibits similar size in the cases of the overall video encoded using the DP tools of the MPEG-4 standard and the low priority layer of the hierarchical video: both being higher than the burst size for the high priority layer. A similar trend is shown in the case of the CDF of the distance between loss bursts, see Figure 8(b). This can be simply explained by realizing that the low priority video represents $91.75 \%$ of the hierarchical video traffic while only the remaining $8.25 \%$ belongs to the high priority video layer. 

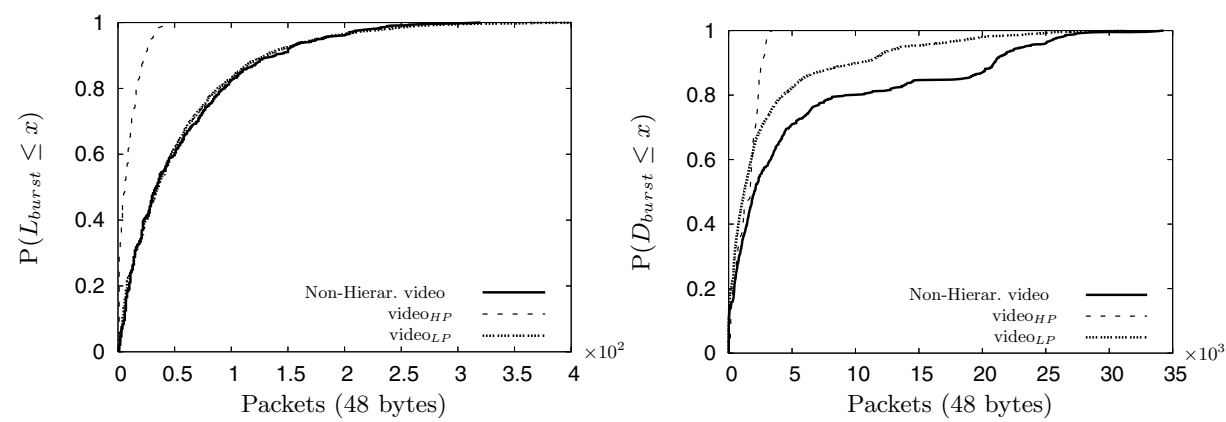

(a) CDF of size of video burstlosses, load (b) CDF of distance between losses video $\approx 100 \%$. burst, load $\approx 100 \%$.

Fig. 8. Pattern of Losses in Hierarchical and non-Hierarchical Video

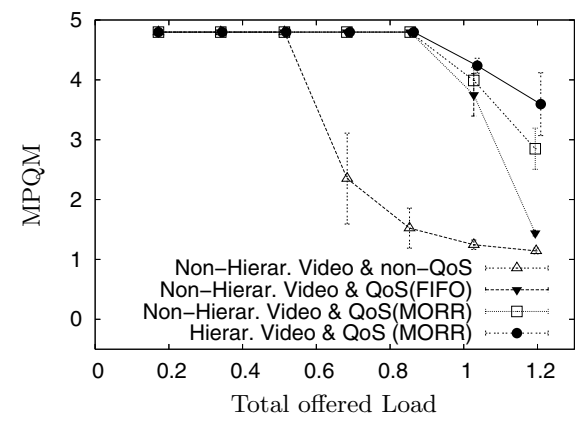

Fig. 9. Quality of Hierarchical and Non-hierarchical Video

To summarize the performance evaluation results, Figure 9 represents the decoded video quality when integrating the different techniques introduced herein. The figure shows that using a resource request algorithm adapted to each type of traffic proves effective for network loads of up to $80 \%$. The figure also shows that the quality can be further improved by using a bandwidth allocation mechanism which minimizes the overhead and making use of a hierarchical video transmission versus a non-hierarchical video transmission.

\section{Conclusions}

In this article, we have evaluated a set of QoS mechanisms and the MPEG4 error resilience tools as a means of deploying effective video services over TDMA/TD wireless networks. Through an extensive campaign of simulations, we have evaluated the capabilities of the overall protocol architecture in terms of various metrics. In particular, we have shown the effectiveness of our proposals in terms of the video quality as perceived by the end-user. 


\section{References}

1. F. Delicado, P. Cuenca, L. Orozco-Barbosa, and A. Garrido, "Design and Evaluation of a QoS-aware Framework for Wireless TDMA/TDD," Wireless Personal Communications Journal, vol. 2005, no. 34, pp. 37-90, 2005.

2. F. Delicado, P. Cuenca, and L. Orozco-Barbosa, "QoS Mechanisms for Multimedia Communications over TDMA/TDD WLANs," to be published in Computer Communications Journal, 2006.

3. Information Technology- Generic Coding of Audio-visual Objects- Part 2: Visual, ISO/IEC Std. 14 496-2, March 1999.

4. F. Delicado, A. Garrido, P. Cuenca, L. Orozco-Barbosa, and F. Quiles, "Improving the Robustness of MPEG-4 Video Communications over Wireless/3G Mobile Networks," in Proc. of 13th IEEE International Symposium on Personal, Indoor and Mobile Radio Communications (PIMRC'02), vol. 4, Lisbon, Portugal, September 2002, pp. $1685-1689$.

5. OPNET Modeler 10.0, (c) 1987-2003 OPNET Technologies, Inc., http://www. opnet.com.

6. Coding of Speech at $16 \mathrm{Kbit} / \mathrm{s}$ Using Low-delay Dode Excited Linear Prediction, ITU-T Std. Rec. G.728, September 1992.

7. G. Colombo, L. Lenzini, E. Mingozzi, B. Cornaglia, and R. Santaniello, "Performance Evaluation of PRADOS: a Scheduling Algorithm for Traffic Integration in a Wireless ATM Networks," in Proc. of ACM MOBICOM'99, Seattle, WA, August 1999, pp. 143-150.

8. A. Klemm, C. Lindemann, and M. Lohmann, "Traffic Modeling and Characterization for UMTS Networks," in Proc. of IEEE GLOBECOM'01, Internet Performance Symposium, San Antonio, TX, November 2001.

9. A. Karam and F. Tobagi, "On the Traffic and Service Classes in the Internet," in Proc. of IEEE GLOBECOM'00, San Francisco, CA, USA, 2000.

10. C. J. Van den Branden and O. Verscheure, "Perceptual Measure Using a SpatioTemporal Model of Human Visual System," Proceedings of SPIE Conference on Electronic Imaging, Digital Video Compression: Algorithms and Technologies, vol. 2668, pp. 450-461, San Jose, January 1996.

11. J. R., A. Durresi, and G. Babic, "Throughput fairness index: An explanation," ATM Forum/99-0045, Tech. Rep., Feb 1999. 